\title{
La pronunciación en inglés para estudiantes de primer año de primaria: una secuencia didáctica para su mejoramiento
}

Por: Mg. Karin Sepúlveda Navarro', Universidad de Concepción, Chile; Mg. Cecilia Quiroga C. ${ }^{2}$, Universidad de Concepción, Chile y Dr. Claudio Díaz L. ${ }^{3}$, Universidad de Concepción, Chile

Recibido: 2 de noviembre, 2017. Aceptado: 11 de marzo, 2018.

\begin{abstract}
Resumen
El presente artículo presenta un estudio realizado con 25 estudiantes de primer año de educación primaria, a los cuales se les se implementó una secuencia didáctica con actividades basadas en el enfoque de "Respuesta Física Total" para mejorar la inteligibilidad en la pronunciación del inglés. En esta secuencia didáctica los estudiantes observaron imágenes y escucharon vocabulario mediante videos y canciones, relacionándolos con movimientos corporales e interactuando a través de breves diálogos. La recolección de datos se obtuvo de un pre-test y post-test de pronunciación oral conformados por tres ítems: repetición de palabras, descripción de objetos y presentación oral, cuyos datos se procesaron mediante un análisis de frecuencia y porcentual. Asimismo, se llevó a cabo un análisis temático para las notas de campo y las entrevistas realizadas. Los resultados reflejaron un mejoramiento en la pronunciación a nivel de palabras, frases y oraciones en inglés, lo que permitió que los participantes produjeran mensajes inteligibles.
\end{abstract}

\section{Abstract \\ English pronunciation for first year primary school students: a didactic sequence implementation for its improvement}

This article presents a study conducted on 25 first-year primary school students. A didactic sequence was implemented utilizing activities that allowed participants to improve their intelligibility in the English pronunciation. The activities were primarily based on the Total Physical Response method for English teaching.

In this didactic sequence the students observed images and listened to vocabulary through videos and songs, relating them to body movements and interacting through brief dialogues. The data collection was carried out with a Pre and Post-Oral Pronunciation test, which included three items: Repetition of Words, Description of Objects, and Oral Presentation; the data obtained was processed by frequency and percentage. Likewise, a thematic analysis was conducted for field notes and interviews. The results revealed an improvement in the pronunciation at word, phrase, and sentence levels in English, and participants achieved an intelligible message.

1 Karin Sepúlveda es Magíster en Educación y labora como profesora de inglés en la Universidad de Concepción, Chile. Contacto: ksepulveda@udec.cl.

2 Cecilia Quiroga es Magíster en Educación y se desempeña como profesora de inglés en la Universidad de Concepción, Chile. Contacto: cquiroga@udec.cl.

3 Claudio Díaz es Magíster en Lingüística y Doctor en Educación, graduado de la Universidad de Concepción, Chile. Se desempeña como profesor de inglés y también como Director del Magíster en Innovación de la Enseñanza, Aprendizaje y Evaluación del Inglés, en esa misma casa de estudios superiores. Contacto: claudiodiaz@udec.cl.
Karin Sepúlveda N., Cecilia Quiroga C. y Claudio Díaz. La pronunciación en inglés para estudiantes de primer año de primaria: una secuencia didáctica para su mejoramiento. Revista Comunicación. Año 39, volumen 27, número 1, enero a junio, 2018. Instituto Tecnológico de Costa Rica. ISSN: 0379-3974 / e-ISSN1659-3820.

\section{PALABRAS CLAVE:}

Lengua extranjera, enseñanza, inteligibilidad, pronunciación, enfoque de Respuesta Física Total, secuencia didáctica.

\section{KEY WORDS:}

Foreign language, teaching, intelligibility, pronunciation, Total Physical Response method, didactic sequence. 


\section{INTRODUCCIÓN}

La enseñanza de una lengua extranjera involucra cuatro habilidades comunicativas: comprensión de lectura, comprensión auditiva, expresión escrita y expresión oral. Esta última involucra diversas variables que influyen en la comunicación e interacción entre hablantes. Una de ellas es la pronunciación, sistema por el cual se entrega un mensaje. Si el mensaje es comprendido, se podrá establecer el intercambio entre hablantes.

La motivación principal de esta intervención surgió a raíz de la observación directa de los discentes, quienes presentaban problemas de inteligibilidad en la pronunciación al expresarse oralmente en inglés. Esto se manifestaba de diferentes maneras en el actuar de los estudiantes como -por ejemplo- en la falta de participación activa en la sala de clases, la bajada de la mirada cuando el profesor les dirigía preguntas relacionadas con la materia, enrojecimiento y silencio. Debido a esto se dio la necesidad de plantear nuevas estrategias didácticas, desde los primeros años de escolaridad, que permitan un aprendizaje significativo y constante, y promuevan el interés y desarrollo lingüístico de los estudiantes, sin perder de vista que la enseñanza de un idioma extranjero requiere un proceso extenso para obtener los resultados deseados por parte de los educandos. El objetivo de esta investigación-acción consistió en implementar una secuencia didáctica sustentada en el enfoque de "Respuesta Física Total" para mejorar la inteligibilidad en la pronunciación en inglés en estudiantes de primer año de educación primaria. Tuvo una duración de cinco sesiones y cada una de ellas constó de dos horas semanales.

Este artículo se inserta en el contexto del proyecto Fondecyt 1150889, titulado Las dimensiones cognitivas, afectivas y sociales del proceso de planificación de aula y su relación con los desempeños pedagógicos en estudiantes de práctica profesional y profesores nóveles de pedagogía en inglés.

\section{MARCO TEÓRICO}

En el proceso didáctico existen diversos factores que influyen en el aprendizaje. En esta ocasión, se hará referencia a aquellos que se considera, deben estar presentes en la enseñanza de un idioma extranjero. Uno de ellos es el ambiente que se vive en una sala de clases. Según Casassus (2008), los ambientes tensos en las escuelas y el hogar atentan contra las capacidades de aprendizaje de los niños. Los ambientes sanos, por su parte, favorecen el equilibrio emocional y consecuentemente, el aprendizaje. Esto da a entender la gran importancia que tiene promover ambientes agradables para los estudiantes, en los cuales ellos se sientan seguros y propicien su desarrollo intelectual y emocional.

El aprendizaje de una lengua es lo que Krashen (2013) define como "un proceso consciente, ya que cuando estamos aprendiendo sabemos que estamos aprendiendo, esto es lo que sucede en la sala de clases" (p. 3). Al aprender una lengua extranjera (L2), los estudiantes comprenden diferentes aspectos relacionados con el vocabulario, la pronunciación y las estructuras gramaticales, los cuales son diferentes a los de la lengua materna. Al respecto, Ortega (2009) afirma que "en cuanto mayor sea la exposición a la lengua más rápido y completo será el aprendizaje" (p. 42). Asimismo, Yilorm (2016) asevera que "es sabido que mientras más sentidos estén incorporados en el proceso de enseñanzaaprendizaje, más se beneficia la apropiación de los saberes" (p. 112).

Vale señalar que las primeras etapas de adquisición de una lengua tienen lugar a través de procesos agradables con el apoyo de la interacción física, acompañados de la visión, habla, expresiones y gestos. Todo esto estimula las respuestas de los niños (Castro y Navarro, 2014). Por su parte, McLaughlin (2012) refiere que "los niños pequeños se acercan a la tarea de aprender la segunda lengua de una manera similar a la que aprenden la lengua materna" (p. 14). Por lo tanto, es necesario partir desde la vivencia de los alumnos mediante alguna experiencia práctica del aspecto que se abordará en los contenidos por tratar (Halbach, 2008).

En la enseñanza de lenguas extranjeras uno de los enfoques más conocidos a nivel mundial es la "Total Physical Response" (TPR), en adelante referida como enfoque de "Respuesta Física Total", creada en 1977 por James Asher, profesor de psicología en la Universidad de San José, California. Al respecto, 
Asher (2003) señala que este enfoque se "caracteriza principalmente por la coordinación del habla y la acción, por el que se intenta enseñar el lenguaje a través de la actividad física" (p. 73). El enfoque de "Respuesta Física Total" trabaja las acciones físicas acompañadas de órdenes, ya que enseña mediante mandatos imperativos y movimientos corporales.

Desarrollar actividades de "Respuesta Física Total" es quizás la herramienta lingüística más útil para un profesor que pretende que los estudiantes adquieran un segundo idioma, ya que las habilidades que se desarrollan se retienen por semanas, meses e incluso años (Asher, 2003). Por su parte, Mastromarco (2004) sostiene que la relación entre el lenguaje y la respuesta física permite la comprensión inmediata de las palabras, afirmando que "la Respuesta Física Total establece una relación entre la causa (el lenguaje) y el efecto (la respuesta física) que hace que el significado de las palabras sea comprendido inmediatamente" (p. 2).

Asher (2003) postula que "la respuesta física del niño señala que el mandato fue comprendido" (p. 56). Es importante considerar que se debe comenzar por mandatos simples como: Stand up, Sit down. Luego se avanza con mandatos más complejos, como: Sit down slowly. Estos "deben ser realizados entre 3 y 4 veces hasta que los estudiantes se sientan preparados para hacerlo por sí mismos" (Muñoz y Valencia, 2011, p. 21). En la enseñanza de una lengua extranjera en el contexto escolar, si la comprensión se ha desarrollado antes de hablar, el éxito en el aprendizaje está garantizado (Asher, 2003). Tal como indican Richards y Rodgers (2002), "los estudiantes hablarán cuando se sientan preparados para hacerlo, a esto se llega cuando han internalizado el lenguaje" (p. 76).

A menudo, cuando los estudiantes aprenden una L2 traen consigo experiencias previas que influyen en la disposición para su aprendizaje. Al respecto Casassus (2008) afirma que "la relación del alumno con la materia a aprender es emocional: le interesa o no le interesa, le gusta o no le gusta, lo afecta o no lo afecta, mientras menos lo afecta muestra menos interés" (p. 89). Por ese motivo es importante propiciar ambientes adecuados, en los cuales se otor- guen herramientas y se utilicen métodos adecuados para facilitar el aprendizaje.

El enfoque de "Respuesta Física Total" permite el desarrollo de una de las actividades preferidas de los niños que, además cobra gran relevancia, especialmente en educación primaria: el juego. Este ha sido utilizado para la diversión en tiempo de ocio, pero los expertos afirman que es un medio idóneo para adquirir conocimientos (Rubio y García, 2013). Para los niños el juego no es solo una herramienta indispensable en el desarrollo físico, cognitivo, psicológico y social, sino también es el mecanismo de aprendizaje más importante en esta etapa de la vida (Delgado, 2015). De acuerdo con Rubio y García (2013) "los juegos ofrecen a los participantes confianza en sí mismos y en sus capacidades" (p. 170).

La pronunciación en la enseñanza de un segundo idioma es el factor que permite a los estudiantes comunicarse de manera más eficaz y rápida con el otro. Por consiguiente, la pronunciación no es solamente portadora de contenido léxico-semántico de las unidades lingüísticas, sino que aporta valor comunicativo de enorme importancia al mensaje, ya que transmite actitudes, intenciones y expresiones (Blanco, 2012). En Chile, una pronunciación inteligible es uno de los aspectos más complicados de dominar en un ambiente escolar, debido a la escasa exposición que existe en el entorno de la gran mayoría de los educandos.

Expertos en la pronunciación, por años han destacado que el mejoramiento de la inteligibilidad es el principal objetivo en la enseñanza de una lengua extranjera (Munro y Derwing, 1995). Los investigadores Munro y Derwing (1995) han realizado diversos estudios relacionados con la inteligibilidad y la definen como "el grado en que se entiende el mensaje del hablante por el oyente" (p. 76). Si no existe una adecuada pronunciación cuando hablamos e interactuamos con los demás, será difícil para el oyente comprender el mensaje que se desea transmitir y de esta manera alcanzar la interacción entre hablantes. Es por ello que es importante exponer a los estudiantes al inglés utilizando los medios más apropiados, valiéndose de enfoques como la "Respuesta Física Total", ya que permite la comprensión y pronunciación del vocabulario relacionándolo 
con movimientos corporales. Asimismo, se refuerza y enfatiza la pronunciación a través de una práctica constante del idioma.

\section{MATERIALES Y MÉTODOS}

El presente proyecto se desarrolló mediante la utilización de la metodología de investigación-acción, puesto que es la que más se adecuaba al tipo de investigación que se deseaba llevar a cabo, incluyendo la participación directa del profesor. Para Kemmis (1988), citado en Latorre (2003), la investigación-acción "es una investigación sobre la práctica realizada por y para los prácticos, en este caso por el profesorado" (p. 25). Este autor refiere -además- que en el ámbito educativo la persona indicada es el mismo docente, ya que es él quien a diario está con los estudiantes y conoce la realidad del medio en el cual se desenvuelve (Latorre, 2003). En la educación es el profesor el que está en contacto directo y continuo con los discentes, conociendo y considerando así aquellas problemáticas que surgen en su realidad educativa y es él quien a su vez ha de innovar en su práctica. Según indica Elliott (2005), "la actividad educativa consiste en la elaboración y experimentación de un proyecto dirigido a facilitar el desarrollo de la comprensión en cada uno de los alumnos que componen el grupo de la

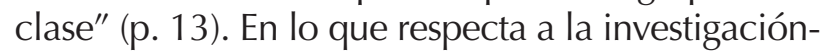
acción en educación, Latorre (2003) afirma que es un proceso que se caracteriza por su espiral cíclico entre la acción y la reflexión, de manera que ambos momentos quedan integrados y se complementan.

\section{OBJETIVOS DEL ESTUDIO}

- Implementar una secuencia didáctica sustentada en el enfoque de la "Respuesta Física Total" para mejorar la inteligibilidad en la pronunciación del inglés en estudiantes de primer año de educación primaria.

- Determinar el impacto de la secuencia didáctica en la pronunciación de los estudiantes a nivel de palabras, frases y oraciones en inglés.

- Identificar la percepción de los participantes sobre la intervención sustentada en el enfoque de "Respuesta Física Total".

\section{SUJETOS DEL ESTUDIO}

Los sujetos del estudio fueron 25 estudiantes, 12 niñas y 13 niños de 5 y 6 años de edad, de primer año de educación básica de un establecimiento de administración particular subvencionada, provenientes de la misma comuna. Todos los estudiantes procedían del mismo establecimiento educativo.

\section{DESCRIPCIÓN DE LA IMPLEMENTACIÓN}

La implementación de este proyecto en estudiantes de primer año de educación básica, cuyo propósito fue mejorar la inteligibilidad de la pronunciación en inglés, está fundamentada en el enfoque de "Respuesta Física Total", con el cual se pretendió que los estudiantes estuviesen expuestos al inglés a través de actividades lúdicas que les motivaran a participar activamente en su proceso de aprendizaje.

Las actividades fueron diseñadas de acuerdo con las necesidades observadas anteriormente, con el fin de desarrollar la pronunciación asociando las palabras a movimientos corporales. En la implementación de la secuencia didáctica sustentada en el enfoque de "Respuesta Física Total" se consideraron factores como el nivel e intereses de los estudiantes. El tema por desarrollar según la unidad didáctica correspondiente a primer año de educación básica fue My toys [Mis juguetes]. Es por ello que dentro del material concreto se consideró el uso de juguetes como apoyo para el desarrollo de la unidad. El presente proyecto se desarrolló en cinco etapas: pre-diagnóstico, diagnóstico, planificación, intervención, evaluación (véase la figura 1).

En la primera etapa de pre-diagnóstico se observó e identificó el problema al cual se deseaba dar solución. Luego, se procedió a adquirir la autorización formal del establecimiento para llevar a cabo el proyecto de investigación-acción. Una vez aceptada la petición, se procedió a solicitar la autorización de los apoderados para la participación de los estudiantes en dicha intervención. Posteriormente, se elaboraron los instrumentos de recogida de información: cuestionario sociodemográfico, test de pronunciación oral, entrevistas sobre la percepción del inglés y rúbricas de desempeño. 
La etapa de diagnóstico constó de dos secciones. En la primera se procedió con los estudiantes, quienes respondieron oralmente una serie de preguntas en español relacionadas con su realidad. Se aplicó -además- un test de pronunciación oral (pre-test de pronunciación), que consistió en tres ítems. En el primero, los estudiantes escucharon y repitieron una serie de palabras mencionadas por un hablante angloparlante. Las palabras repetidas fueron grabadas para su posterior análisis. En el segundo ítem, los estudiantes observaron útiles escolares, escogieron tres de ellos y los describieron, siendo nuevamente grabados. En el tercer ítem, los estudiantes expusieron oralmente una presentación relativa a la unidad de la familia. El fin de esta sección fue obtener información relacionada con el nivel de palabras, frases y oraciones que el educando podía mencionar en inglés.

En la etapa de planificación, de acuerdo con los resultados obtenidos en la etapa de diagnóstico, se programó una cantidad de sesiones, distribuyendo las actividades de la manera más adecuada posible para alcanzar los objetivos establecidos en la investigación, así como los materiales y recursos necesarios con el propósito de satisfacer las necesidades de los pequeños educandos. Luego de esto se procedió a la elaboración de las actividades basadas en el enfoque de "Respuesta Física Total", las cuales serían utilizadas en la etapa de intervención, ya que ellas promueven una participación activa por parte de los estudiantes al realizar movimientos corporales relacionados directamente con el vocabulario de la unidad trabajada, lo que promueve la retención y reconocimiento de este. Además, se consideró el uso de grabaciones de los estudiantes y notas de campo, ya que estas permitirían una observación directa de aquello que ocurría en el proceso mismo de la intervención.

Durante la etapa de intervención se llevaron a cabo cinco sesiones de 90 minutos. Cada una estaba constituida por una estructura similar en la que se comenzaba con el saludo mediante un canto de bienvenida, con lo que se daba inicio a la clase. Después se recordaba el vocabulario de la sesión previa, para lo que se utilizaba realia [objetos reales]. Posteriormente, se observaba un video relacionado con el entorno de los estudiantes y el vocabulario que se desarrollaría en la unidad. Seguidamente, se procedía al uso del enfoque de "Respuesta Física Total". Los estudiantes escuchaban y observaban movimientos corporales realizados por el profesor al mencionar uno de los juguetes de la unidad. Estos movimientos eran imitados por los discentes, luego repetidos oralmente.

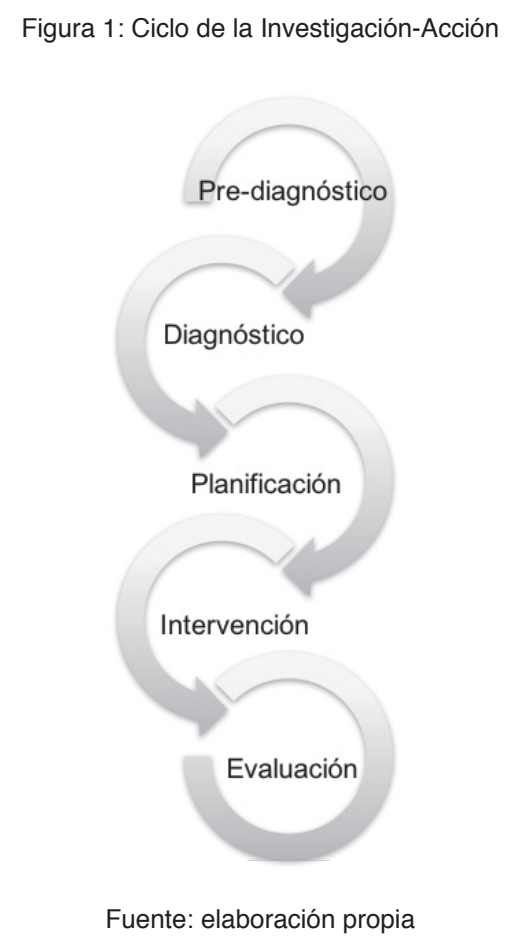

En una primera instancia, los estudiantes observaban para después dar la posibilidad, a uno o dos voluntarios, de pasar frente a la clase y pedir al resto de sus compañeros reaccionar físicamente a los mandatos que ellos proponían, así como mencionar los juguetes según lo que observaban por parte de sus compañeros. De esta manera, los discentes participaban en la clase, generando también un clima más agradable para disfrutar y aprender en conjunto con sus pares. Asimismo, se utilizaban canciones, interacción entre pares, especialmente en la realización de las actividades que incluyeran la producción de breves diálogos. Al término de cada sesión los estudiantes resumían lo que habían desarrollado y mencionaban lo aprendido. Finalmente, se procedía a la despedida. De esta forma existía una claridad en lo que correspondía al término de la clase. Al finalizar la intervención, se aplicó el post-test de pronunciación que contenía la misma estructura del pre-test 
de pronunciación, modificando solamente el contenido; esto con el objetivo de comparar los resultados obtenidos en ambas etapas de la investigación.

Una vez recolectada la información, se reflexionó y analizó en torno a los resultados conseguidos, ya que de acuerdo con ellos se pueden diseñar actividades para adaptar y mejorar las acciones llevadas a cabo, con el fin de que los estudiantes alcancen mejores aprendizajes en cuanto a la enseñanza del inglés.

\section{INSTRUMENTOS DE RECOGIDA DE LA INFORMACIÓN}

\section{Test de pronunciación oral (pre y post-test de pro-} nunciación) (véase el Anexo 1)

Con el propósito de obtener la información necesaria sobre el nivel de inteligibilidad de los estudiantes en inglés, se aplicó un pre-test de pronunciación en inglés grabado individualmente en formato mp3. El test fue organizado en tres ítems, en los cuales se evaluaba a los estudiantes a nivel de palabra, frase y oración en inglés.

\section{Rúbrica analítica de desempeño de repetición de palabras (véase el Anexo 2)}

La rúbrica analítica de desempeño de repetición de palabras constaba de un criterio que abordaba la repetición de palabras y tres niveles de desempeño: Adecuado, Suficiente e Insuficiente, lo que sumaba un total de 15 puntos. La pronunciación de palabras fue transcrita individualmente y contaba con el modelo de pronunciación ideal y la pronunciación del estudiante. Cabe señalar que esta rúbrica fue utilizada al inicio del proyecto en la etapa de diagnóstico y luego de la intervención. La única modificación surgió en la sección de transcripción de palabras, ya que eran diferentes a las evaluadas en el pre-test de pronunciación.

\section{Rúbrica analítica de desempeño de descripción de objetos}

La rúbrica analítica de desempeño de descripción de objetos constaba de un criterio que buscaba evaluar la inteligibilidad de las frases de los estudiantes al describir tres objetos y contaba con tres niveles de desempeño: Adecuado, Suficiente e Insuficiente. Las oraciones producidas por los estudiantes fueron posteriormente transcritas en el mismo documento. Se utilizó la misma rúbrica de desempeño para el pre-test y post-test de pronunciación.

\section{Rúbrica analítica de desempeño en la presentación oral}

La rúbrica analítica de desempeño en la presentación oral contenía igualmente un criterio cuyo objetivo principal era obtener el nivel de inteligibilidad de los estudiantes al producir oraciones mediante una presentación oral. Los niveles de desempeño considerados fueron cuatro: Excelente, Bueno, Suficiente e Insuficiente y al igual que las demás rúbricas, esta fue utilizada para analizar el pre-test y post-test de pronunciación.

\section{Notas de campo}

El instrumento de observación utilizado en la etapa de seguimiento fue el de notas de campo, las cuales consideraron la descripción detallada de las reacciones de los estudiantes durante las actividades desarrolladas en las cinco sesiones de la intervención. Estas reflejaron de la forma más fiel y objetiva posible lo acontecido en la sala de clases.

\section{PROCEDIMIENTO DE APLICACIÓN DE LOS INSTRUMENTOS}

Test de pronunciación oral (pre-test de pronunciación)

El pre-test de pronunciación fue aplicado antes de la intervención y constaba de tres ítems. En el primero, los estudiantes repetían palabras aisladas luego de escuchar la palabra grabada en un diccionario en formato digital. En el segundo ítem, los estudiantes escogían tres objetos que estaban en la mesa del profesor y los describían. En el tercer ítem los estudiantes realizaron una presentación oral. Los primeros dos ítems fueron grabados durante el primer bloque (8:30 a.m.), con una duración aproximada de 45 minutos, mientras que el tercero, una presentación oral, tuvo lugar en el segundo bloque (9:15 a.m.), con una duración de 30 minutos aproximadamente. 


\section{Notas de campo}

Las notas de campo fueron tomadas en un cuaderno al finalizar cada una de las sesiones correspondientes. En ellas se apuntaba el día, la hora, cantidad de participantes, así como la distribución de la sala de clases y lo más importante, la reacción de los estudiantes frente a las actividades que desarrollaron en la intervención.

\section{Test de pronunciación oral (post-test de pronuncia- ción oral)}

El post-test de pronunciación fue aplicado luego de la intervención, el cual contenía la misma estructura del pre-test de pronunciación. Este tuvo lugar en la sala de clases. En el primer bloque se aplicaron los dos primeros ítems, con una duración aproximada de 45 minutos. El tercer ítem tuvo una duración de alrededor de 30 minutos. En esta etapa fue posible evaluar a la mayoría de los estudiantes. Hubo solo tres que no asistieron a clases por problemas de salud. Ellos fueron evaluados en la siguiente clase.

\section{PROCEDIMIENTOS DE ANÁLISIS DE LA INFORMACIÓN}

La interpretación de la información obtenida se realizó mediante el análisis de frecuencia y porcentual. Se procedió primeramente a la transcripción de cada una de las rúbricas utilizadas: rúbrica analítica de desempeño de repetición de palabras, rúbrica analítica de desempeño de descripción de objetos, rúbrica analítica de desempeño en la presentación oral. Luego, de la transcripción de los datos, estos fueron traspasados a formato .XLSX (Excel) y se elaboraron gráficos para su posterior análisis. El proceso de seguimiento se llevó a cabo por medio de notas de campo en las que se llevó a cabo una observación descriptiva que permitió la realización de un análisis temático de las clases observadas.

\section{RESULTADOS}

Los resultados obtenidos en ambas evaluaciones, previo y posterior a la intervención, han demostrado que los estudiantes de primer año básico mejoraron notablemente su nivel de inteligibilidad, así como el vocabulario adquirido en la unidad desarrollada.
A continuación se presentan gráficos comparativos de las evaluaciones, en los cuales los primeros datos corresponden a los niveles alcanzados en la etapa de diagnóstico y los segundos a aquellos obtenidos después de la intervención.

En la figura 2 se observan los resultados obtenidos por los estudiantes en el primer ítem del test de repetición de palabras. En el pre-test de pronunciación, repetición de palabras, solo 16\% de los participantes alcanzó un nivel adecuado, mientras que en el post-test del mismo ítem casi la totalidad de los estudiantes alcanzó 96\% de logro. Esto muestra que la inteligibilidad de la pronunciación de los estudiantes se incrementó alrededor de 80\%.

Por otra parte, en lo que respecta al nivel Suficiente, en el pre-test de pronunciación fue alcanzado por $48 \%$ de los estudiantes y solo $4 \%$ en el mismo nivel del test aplicado posterior a la intervención (ver figura 2). Se debe destacar, además, que luego de la intervención ninguno de los estudiantes obtuvo un puntaje correspondiente al nivel Insuficiente. Los resultados reflejan un aumento considerable en el nivel de logro relacionado con la pronunciación de palabras aisladas.

Figura 2. Nivel de desempeño alcanzado en repetición de palabras

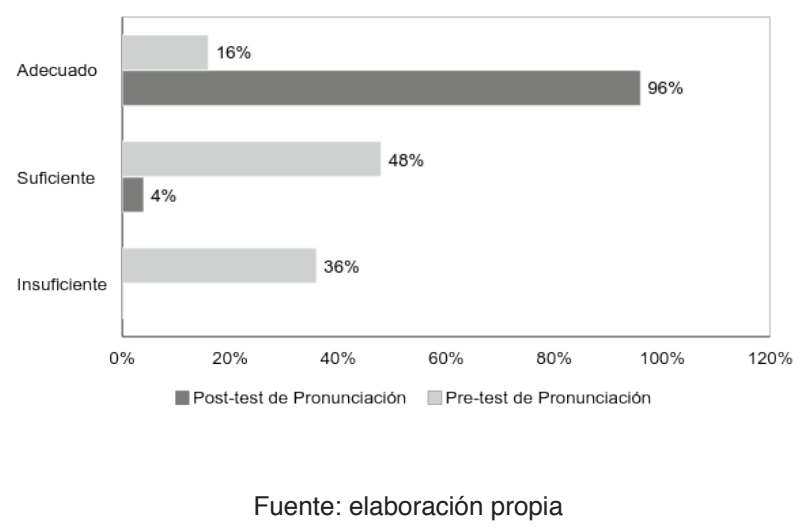

La figura 3, correspondiente a los resultados del segundo ítem, descripción de objetos, muestra un aumento en el nivel de desempeño Adecuado y Suficiente. En el pre-test de pronunciación 44\% se ubicó en el nivel Adecuado, mientras que en el posttest de pronunciación $60 \%$ en la misma sección. El desempeño de los estudiantes en esta sección aumentó en 16\%. En lo que refiere a un desempeño 
Suficiente, disminuyó el porcentaje en 12\%. Los estudiantes mejoraron su nivel de desempeño y al igual que en el ítem anterior, ninguno obtuvo un nivel que lo ubicara en el nivel Insuficiente. Estos resultados arrojan nuevamente un avance positivo en cuanto a la descripción de objetos (véase la figura 3).

Figura 3. Nivel de desempeño alcanzado en descripción de objetos

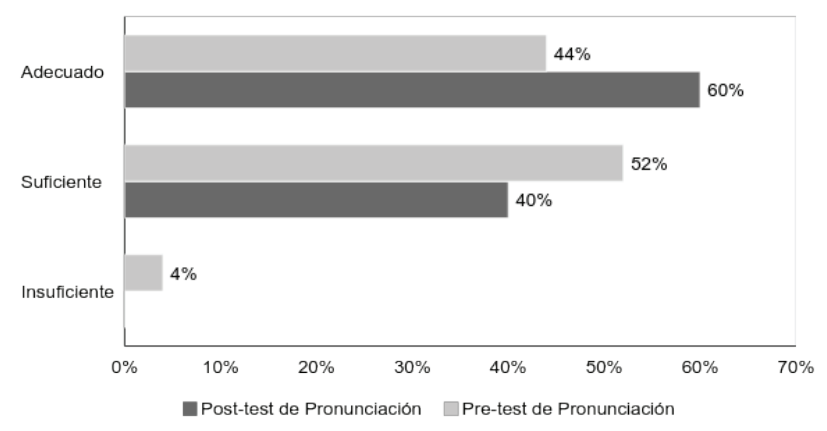

Fuente: elaboración propia

En la figura 4 se puede observar que en el último ítem del test de pronunciación, correspondiente a la presentación oral, el nivel de desempeño alcanzado por la mayoría de los estudiantes tuvo un incremento destacable en el mensaje transmitido. La casi inexistencia de errores conlleva a que lo expresado oralmente fuese completamente inteligible (véase la figura 4). Los porcentajes en el inicio de la investigación en el mayor nivel de rendimiento fueron alcanzados solo por $16 \%$ de los estudiantes, mientras que -después de la etapa de intervencióneste aumentó en $40 \%$. Vale destacar que -además de la inteligibilidad del mensaje- las oraciones emitidas fueron más completas y elaboradas, como lo refleja el participante 4: Hello, my name is Emilio. My robot is black, my ball is red, my hoop is green, my teddy is orange... En la primera presentación este participante no deseaba realizar la presentación oral, porque tenía vergüenza al no recordar las palabras ni su significado, por lo que manifestó: "No sé cómo, mamá. No recuerdo en inglés. Se me olvidó".

En lo concerniente a los niveles más bajos -Suficiente e Insuficiente- disminuyeron notoriamente. En el primero existe una disminución de 12\% con relación a la primera etapa y en el segundo ninguno de los participantes se posicionó en el nivel más bajo de desempeño. Los porcentajes relacionados con el rendimiento de los educandos en cada uno de los ítems demuestran que hubo un aumento considerable en lo que respecta la inteligibilidad de los mensajes emitidos luego de haber sido intervenidos mediante la implementación del enfoque de "Respuesta Física Total".

Figura 4. Nivel de desempeño alcanzado en presentación oral

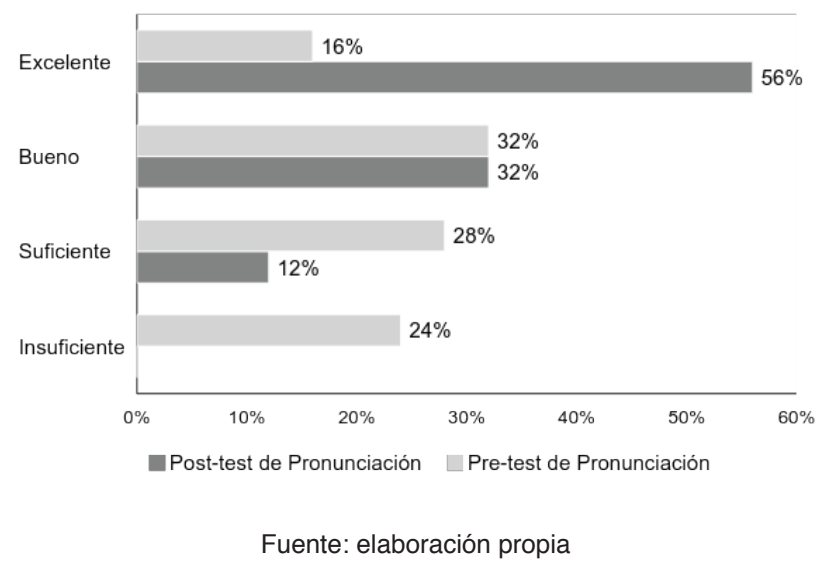

Al inicio y término de la investigación se llevó a cabo una entrevista de percepción sobre la asignatura de inglés. Al comparar los resultados de ambas entrevistas que -a pesar de no contener las mismas preguntas- apuntaban a un mismo objetivo, que era conocer el punto de vista de los estudiantes con respecto al inglés en el contexto escolar, los resultados de estas entrevistas mostraron que luego de la intervención los estudiantes tenían una reacción completamente positiva hacia el inglés. En las respuestas de la segunda entrevista, la mayoría de los estudiantes manifestó que le agradó todo lo que hicieron en las últimas sesiones. Los dos estudiantes que respondieron de forma negativa hicieron referencia específicamente a los objetos seleccionados, ya que estos no les identificaban.

En la primera entrevista dos estudiantes manifestaron que no les gustaba hablar en inglés, porque era muy difícil (participante 17) o porque solo sabían hablar en español (participante 24). En la segunda entrevista, luego de la intervención, los mismos estudiantes dijeron: "todo me gustó", "nada no me gustó". En lo que respecta a las actividades que 
contestaron les agradaban, se puede señalar que los estudiantes disfrutan definitivamente de actividades en las cuales puedan moverse, interactuar o manipular objetos jugando. En la primera entrevista ellos evidenciaron el gusto por jugar con sus pares y en la pregunta número dos -que se refiere a lo que más les gustó de las últimas clases de inglés- se repitió en reiteradas ocasiones jugar con los juguetes.

Las respuestas obtenidas y posteriormente comparadas demuestran que la actitud y percepción con respecto a la asignatura tuvo una mejora destacable respecto de la primera impresión conseguida en la etapa previa a la intervención. Mediante la implementación de actividades sustentadas en el enfoque de "Respuesta Física Total", los estudiantes -especialmente aquellos que mostraban una actitud negativa frente al inglés- modificaron su percepción, expresando el gusto por aprender este idioma mediante las diversas actividades desarrolladas.

Para concluir con la comparación de los resultados recopilados al inicio y término de la intervención y al observar los análisis recientemente descritos, se puede confirmar fehacientemente que el uso del enfoque de "Respuesta Física Total" permitió que los estudiantes mejoraran su nivel de desempeño y adquisición del idioma inglés casi por completo. Sus resultados destacables hacen ver que cuando se utilizan los métodos adecuados al nivel de desarroIlo de los estudiantes -considerando sus intereseses posible que estos mejoren su rendimiento académico sin dejar de lado la reacción positiva hacia la lengua extranjera, que es -sin duda alguna- uno de los principales factores en el aprendizaje, ya que aprendieron desde su propia experiencia, relacionando la expresión oral con los movimientos, al igual que los objetos con su realidad.

En lo que respecta al seguimiento de la intervención, esta etapa se llevó a cabo mediante el instrumento de notas de campo, lo que permitió reflejar la reacción de los estudiantes frente a las actividades desarrolladas durante las cinco sesiones. De acuerdo con los datos obtenidos, se observaron los siguientes resultados, mostrados en la figura 5, que ilustra la reacción de los estudiantes respecto de las sesiones llevadas a cabo en este proyecto de investigación-acción.
En las primeras sesiones, especialmente en la primera, los participantes observaron con sonrisas y rostros sorprendidos los juguetes en la mesa del profesor; esto los llevó a preguntar: "Miss, ¿para qué son los juguetes?". Con esta interrogante se dio inicio a la unidad. Todos los discentes miraron y escucharon atentamente los comentarios del profesor y respetaron el turno para dar su opinión respecto de los juguetes, procurando a su vez imitar las palabras que escuchaban en inglés. Al terminar la clase, los estudiantes se mostraron muy entusiasmados, expresando su satisfacción mediante comentarios como: "Me gustó la clase" o "A mí me gusta jugar con el teddy".

Asimismo, los estudiantes se mostraron cómodos al desarrollar las actividades relacionadas con movimientos corporales, expresión oral y breves tareas asignadas en la clase. Imitaron los movimientos que observaron y escucharon con las canciones intentando seguir su melodía y las palabras. En la última actividad de la primera sesión colorearon sus juguetes favoritos y los recortaron, pegándolos luego en un trozo de cartulina. El tiempo que les tomó realizar esta tarea fue el planificado y la desarroIlaron dialogando y compartiendo sus materiales. A su vez, en la tercera sesión -al observar un video relacionado con los juguetes- uno de los estudiantes -cuando vio la pelota- se puso de pie e imitó el movimiento mencionando el objeto. Luego de esto los demás siguieron su ejemplo y llevaron a cabo los movimientos correspondientes (véase la figura 5).

La figura 6 muestra que casi la totalidad de los estudiantes tuvo una participación activa en la mayoría de las actividades, especialmente en la primera sesión, en la que los educandos manipularon los juguetes. Algunos jugaron con el auto, otros tomaron la pelota, la dejaron en el suelo y realizaron breves movimientos con los pies. En voz baja algunos indicaron al compañero "Toma la pelota, pero con el pie" [sonrieron]. Sin embargo, en el inicio de esta misma sesión, la actividad en la que imitaron los movimientos del profesor relacionándolo con un objeto, se pudo observar que dos estudiantes, los más tímidos, no ejecutaron ningún movimiento, solo observaron a sus compañeros y al profesor. No obstante, luego de mirar a sus pares con rostros 
sonrientes y moviéndose libremente, comenzaron a esbozar sonrisas y posteriormente imitaron los movimientos.

\begin{tabular}{l} 
Figura 5. Reacción de los estudiantes en \\
las actividades desarrolladas \\
\begin{tabular}{|l|c|l|}
\hline $\begin{array}{c}\text { Reacción } \\
\text { de los estu- } \\
\text { diantes }\end{array}$ & $\begin{array}{c}\text { Cantidad } \\
\text { de estu- } \\
\text { diantes }\end{array}$ & \multicolumn{1}{|c|}{ Comentarios } \\
\hline Positiva & 23 & $\begin{array}{l}\text { En todas las sesiones } \\
\text { los estudiantes mostra- } \\
\text { ron rostros sonrientes } \\
\text { y sorprendidos frente } \\
\text { a las actividades desa- } \\
\text { rrolladas. }\end{array}$ \\
\hline Negativa & 2 & $\begin{array}{l}\text { En la primera sesión } \\
\text { los estudiantes más } \\
\text { tímidos no participa- } \\
\text { ron desde el inicio de } \\
\text { la clase, manifestando } \\
\text { no saber lo que se de- } \\
\text { bía hacer. }\end{array}$ \\
\hline Total & 25 & \\
\hline
\end{tabular} \\
\hline
\end{tabular}

La escasa participación de estos estudiantes fue completamente diferente en la segunda sesión, ya que en el inicio de la clase fueron precisamente ellos quienes levantaron su mano pidiendo pasar al frente de la clase para realizar los movimientos relacionados con los juguetes. Los más aventajados y con mayor personalidad se pusieron de pie para mostrar lo que habían aprendido, pero se autorregularon y respetaron su turno para poder participar. Asimismo, en las últimas sesiones la totalidad de los estudiantes participaron activamente en el intercambio de breves diálogos en los que describieron sus juguetes favoritos, manifestando así sus preferencias y respetando además su turno para hablar (ver figura $\mathrm{N}^{\mathrm{o}}$ 6). No obstante, en la sesión en la que se procedió a la repetición de palabras hubo tres estudiantes que en un principio estuvieron en silencio, período que para algunos fue necesario. Una vez que comprendieron el vocabulario, comenzaron a participar activamente repitiendo las palabras. Esta actitud se prolongó durante las sesiones precedentes.
Figura 6. Participación de los estudiantes en las actividades

\begin{tabular}{|c|c|c|}
\hline Actividades & $\begin{array}{l}\text { Cantidad } \\
\text { de estudi- } \\
\text { antes }\end{array}$ & Comentarios \\
\hline $\begin{array}{l}\text { Movimien- } \\
\text { tos corpo- } \\
\text { rales }\end{array}$ & 23 & $\begin{array}{l}\text { Solo dos estudiantes, } \\
\text { los más tímidos, no } \\
\text { participaron activa- } \\
\text { mente en el inicio de } \\
\text { la intervención, en la } \\
\text { realización de mov- } \\
\text { imientos. }\end{array}$ \\
\hline $\begin{array}{l}\text { Repetición } \\
\text { de palabras }\end{array}$ & 22 & $\begin{array}{l}\text { En esta sección que - } \\
\text { al parecer era la más } \\
\text { compleja- solo tres } \\
\text { estudiantes se mantu- } \\
\text { vieron en silencio, sin } \\
\text { repetir las palabras. } \\
\text { Esta actitud cambió en } \\
\text { la segunda sesión. }\end{array}$ \\
\hline $\begin{array}{l}\text { Manipu- } \\
\text { lación de } \\
\text { objetos }\end{array}$ & 25 & $\begin{array}{l}\text { Todos los estudiantes } \\
\text { manipularon los obje- } \\
\text { tos. Algunos jugaron } \\
\text { con ellos. }\end{array}$ \\
\hline $\begin{array}{l}\text { Interacción } \\
\text { con los } \\
\text { pares }\end{array}$ & 25 & $\begin{array}{l}\text { La totalidad de los es- } \\
\text { tudiantes interactuó } \\
\text { con sus compañeros } \\
\text { en un breve diálogo } \\
\text { por medio del que } \\
\text { manifestaron sus in- } \\
\text { tereses. }\end{array}$ \\
\hline
\end{tabular}

Fuente: elaboración propia

En la figura 7 se observa que el ambiente de la sala de clases era agradable. Los estudiantes mostraron sentirse libres y contentos al realizar movimientos y manipular los objetos, compartiendo e interactuando con sus pares. En la mayoría de las sesiones (4 de 5), los estudiantes se mostraron alegres en las diversas actividades desarrolladas durante la intervención (ver figura 7).

Vale destacar que en la primera sesión se escucharon risas a carcajadas debido a un error que cometió el profesor al querer dominar la pelota, ya que -en lugar de dominarla- esta golpeó la puerta de la sala. Algunos estudiantes comenzaron a reírse de 
inmediato, mientras el resto observaba al profesor esbozando leves sonrisas. Seguido de este desacierto por parte del docente, un estudiante intentó dominar el hula-hula con la cintura, pero no lo logró. Optó por hacerlo girar en su brazo, exclamando: "¡También se puede con el brazo!" y sonrió. Lo descrito vislumbra que el humor fue parte integradora de la sala de clases, propiciando desde el principio un ambiente atractivo para los estudiantes.

Sin embargo, en la penúltima sesión esta situación cambió debido a la presencia del profesor tutor en la sala de clases, ya que -al notar su presenciahubo estudiantes que le observaban bajando su mirada, sin participar activamente de las actividades que se desarrollaron mientras este permaneció en el aula. Esta actitud dio a entender que se sentían incómodos con su presencia. En síntesis, el ambiente percibido en casi la totalidad de las sesiones fue agradable para los estudiantes. Esto se manifestó en la participación activa por parte de ellos. El humor, la confianza y la relación entre pares, como con el profesor, permitieron que existiera un ambiente propicio para un aprendizaje significativo.

Figura $N^{\circ} 7$. Ambiente percibido en la sala de clases

\begin{tabular}{|c|c|l|}
\hline Percepción & Sesiones & \multicolumn{1}{|c|}{ Comentarios } \\
\hline Agradable & 4 & $\begin{array}{l}\text { En la mayoría de las } \\
\text { sesiones los estudian- } \\
\text { tes parecieron sentirse } \\
\text { cómodos, participando } \\
\text { activamente en las ta- } \\
\text { reas encomendadas. }\end{array}$ \\
\hline Desagradable & 1 & $\begin{array}{l}\text { En la penúltima sesión } \\
\text { se observaron rostros } \\
\text { que reflejaron timidez } \\
\text { con la presencia del } \\
\text { profesor tutor. Bajaron } \\
\text { la mirada, no hablaron } \\
\text { ni participaron durante } \\
\text { su presencia en la sala } \\
\text { de clases. }\end{array}$ \\
\hline
\end{tabular}

Fuente: elaboración propia

\section{DISCUSIÓN Y CONCLUSIONES}

Una vez finalizada la intervención de esta investigación-acción en la asignatura de inglés, fue posible constatar -por medio de los resultados obtenidosque la implementación fue la adecuada, ya que los niveles de logro alcanzados por los estudiantes fueron los esperados.

El uso del enfoque de "Respuesta Física Total" permitió que los alumnos adquirieran un mayor nivel de inteligibilidad, entregando un mensaje comprensible para el interlocutor, lo que promovía el movimiento corporal, producción de palabras y mensajes en inglés, con lo que se obtuvo una recepción positiva por parte de los estudiantes, respetando la etapa de desarrollo, sus intereses y motivaciones.

En el proceso de intervención se pudo confirmar la participación activa de los estudiantes en cada una de las sesiones, cumpliendo con los tiempos planificados (Muñoz y Valencia, 2011). Cuando los estudiantes expresaron abiertamente que deseaban manipular los juguetes, se coincidió con lo que afirman Rubio y García (2013) en cuanto al uso de materiales auténticos, ya que aumentó el nivel de implicación de los niños, incluso de los estudiantes más tímidos.

Las reacciones positivas de este grupo de estudiantes pueden dar cuenta de lo que señala Mastromarco (2004) respecto a la "Respuesta Física Total", ya que se estableció una relación entre el lenguaje y la respuesta física, lo que hizo que el significado de las palabras fuera comprendido inmediatamente. La ejecución de movimientos corporales demandó por parte de los estudiantes la comprensión de lo que realizaron disfrutando, lo que hacían sin necesidad de comprender la totalidad del vocabulario.

Es importante mencionar que los estudiantes a esta edad son bastante activos. Fue por ello que se decidió, acudiendo a los hallazgos de diversos investigadores en el área, implementar una secuencia de actividades fundamentada en el enfoque de "Respuesta Física Total", ya que promovió la participación activa de los estudiantes. Según señala Harmer (2007). "al ser niños su comprensión no proviene sólo de la explicación, sino también de lo que ellos ven y escuchan, con 
lo que tocan o con quien interactúan" (p. 82). Una vez adquirido el movimiento relacionando la acción con lo que escuchaban, se pudo pasar al reforzamiento de la pronunciación de palabras en inglés. No se forzó a los estudiantes a producir las palabras, ya que -como bien señalan Richards y Rodgers (2002) - "los estudiantes hablarán cuando se sientan preparados para hacerlo, a esto se llega cuando han internalizado el lenguaje" (p. 76).

Los resultados analizados demostraron que las actividades elaboradas para abordar las necesidades que presentaban los estudiantes en la pronunciación del inglés fueron las adecuadas. En relación con el impacto de la secuencia didáctica en la pronunciación de los estudiantes, los resultados comprobaron el mejoramiento en todos los ítems evaluados. En el primer ítem del test, la inteligibilidad de los discentes en la pronunciación, al repetir palabras aisladas se incrementó en $80 \%$ el nivel Adecuado luego de la intervención. Por otra parte, el porcentaje de estudiantes en el nivel Suficiente disminuyó en $44 \%$ respecto del pre-test de pronunciación. Además, es necesario señalar que posterior a la intervención, ninguno de los estudiantes se mantuvo en el nivel de desempeño Insuficiente.

En lo que respecta al segundo ítem del post-test de pronunciación, se observó un incremento de 16\% en el nivel Adecuado. Los resultados en este ítem manifestaron nuevamente un avance positivo en cuanto a la descripción de objetos. En el tercer y último ítem, los estudiantes obtuvieron un desempeño Destacable en la presentación oral. Su mensaje fue inteligible, aumentando su rendimiento en $40 \%$ en el nivel más alto de desempeño y al igual que en los ítems anteriores, ninguno de los estudiantes se ubicó en el nivel más bajo de desempeño. Mediante la secuencia de actividades implementada en esta investigación se logró un impacto positivo en los estudiantes, ya que superaron notablemente los niveles de desempeño alcanzados en la etapa de diagnóstico, mejorando claramente su pronunciación, además de adquirir nuevo vocabulario. Una vez finalizada la intervención, en la entrevista sobre la percepción del inglés, que tuvo relación directa con la opinión de los estudiantes acerca de la implementación del enfoque de "Respuesta Física total", estos expresaron que poseían una percepción positiva respecto de la enseñanza de este idioma en el contexto educativo.

Lo expuesto anteriormente demuestra que los objetos reales y cercanos a su entorno motivaron la participación activa desde el principio de la intervención, con los juguetes en sus manos fue posible apreciar la relación que los niños tienen con estos objetos, reflejándose en sus rostros sonrientes y la interacción que surgía al intercambiarlos, jugar con ellos por un momento mediante actividades lúdicas propiciaba un ambiente agradable, ideal para lograr aprendizajes significativos. La implementación del enfoque de "Respuesta Física Total" indudablemente permitió que los estudiantes aprendieran y pronunciaran las palabras de una manera más inteligible, puesto que promovió su respuesta mediante movimientos corporales. Para concluir, este proyecto de investigación-acción implicó que los estudiantes tuvieran la oportunidad de conocer una nueva forma de aprender un idioma extranjero mediante el enfoque de "Respuesta Física Total", que implicaba la participación activa en su propio aprendizaje, en el que la enseñanza de un idioma puede ser activa e interactiva dejando de lado el cuaderno, práctica a la que están habituados.

Al identificar los problemas que se presentan en la sala de clases, estos se pueden resolver utilizando los medios adecuados de acuerdo a la etapa e intereses de los estudiantes, ya que si se desea un cambio en la educación que vaya en beneficio de los que están en este proceso de aprendizaje, es necesario modificar las prácticas docentes.

\section{REFERENCIAS BIBLIOGRÁFICAS}

Asher, J. (2003). Organizing Your Classroom for Successful Second Language Acquisition. Recuperado de http://citeseerx.ist.psu.edu/viewdoc/do wnload?doi=10.1.1.549.2429\&rep=rep 1 \&type $=$ pdf.

Blanco, A. (2012). Corpus oral para el estudio de la adquisición y aprendizaje del componente fónico del español como lengua extranjera. RLA, Revista de Lingüística Teórica y Aplicada, 50 (2), 13-37. Recuperado de 
La pronunciación en inglés para estudiantes de primer año de primaria: una secuencia didáctica para su mejoramiento

http://www.scielo.cl/scielo.php?script=sci_artte xt\&pid=S0718-48832012000200002.

Casassus, J. (2008). Aprendizajes y emociones clima en el aula. Paulo Freire, Revista de Pedagogía Crítica, 7 (6), 81-95. Recuperado de http:// www.uca.edu.ar/uca/common/grupo18/files/ Aprendizaje_emociones_y_clima_de_aula.pdf.

Castro, I. y Navarro, L. (2014). The Role of Songs in First-Graders. "Oral Communication Development in English. PROFILE Issues in Teachers" Professional Development, 16 (1), 11-28. Recuperado de http://www.scielo. org.co/scielo.php?script=sci_abstract\&pid $=$ S1657-07902014000100002.

Delgado, I. (2015). El juego infantil y su metodología. Recuperado de https://books.google. $\mathrm{cl} /$ books?hl=es\&lr=\&id=sjidLgWM9_8C\&o $\mathrm{i}=\mathrm{fnd} \& p g=\mathrm{PA} 2 \& \mathrm{dq}=\mathrm{importancia}+\mathrm{del}+\mathrm{jueg}$ $\mathrm{O}+$ en+la+interacci $\% \mathrm{C} 3 \% \mathrm{~B} 3 \mathrm{n}+$ infantil\&ots= xEHE7vLL64\&sig=dtH2FUYfS7n9qcyeavvti HF_q_4\#v=onepage \&q=interaccion $\% 20$ social $\% 20 y \% 20$ comunicacion $\& f=$ false.

Elliott, J. (2005). La investigación-acción en educación. Recuperado de https://books.google.cl/ books?hl=es\&|r=\&id=eG5xSYGsdvAC\&oi=fn $\mathrm{d} \& p g=$ PA9\&dq $=$ Elliott $+1988+$ Investigacion $+\mathrm{a}$ ccion \&ots $=q$ T91 dob2q6\&sig $=$ AhQelldNTMs JlkKGjT--hu1 uXxE\#v=onepage \&q=Elliott\%20 $1988 \% 20$ Investigacion\%20accion \&f=false.

Halbach, A. (2008). Una metodología para la enseñanza bilingüe en la etapa de Primaria. Revista de Educación, 346 (1), 455-466. Recuperado de http://www.ince.mec.es/revistaeducacion/ re346/re346_17.pdf.

Harmer, J. (2007). The Practice of English Language Teaching. Recuperado de https://www.academia.edu/25472823/The_Practice_of_English_ Language_Teaching_4th_Edition_-_Jeremy_ Harmer.

Krashen, S. (2013). Second Language Acquisition: Theory, applications, and some conjectures. Recuperado de http://www.sdkrashen.com/content/articles/krashen_sla.pdf.
Latorre, A. (2003). La investigación-acción. Conocer y cambiar la práctica educativa. Barcelona: Editorial GRAÓ, de IRIF, S.L.

Mastromarco, A. (2004). Learning Italian at School with TPR by Playing, Creating, and Doing. Recuperado de http://www.tprworld.com/mm5/ merchant.mvc?Screen=CTGY \&Category Code $=200$.

McLaughlin, B. (2012). Second-Language acquisition in Childhood. Recuperado de https:// books.google.cl/books? hl=es\&lr=\&id=YdD XAQAAQBAJ\&o $i=f n d \& p g=P R 1 \& d q=a g e+$ and +secong+language+acquisition \&ots $=D$ 8A14vAg97 \&sig=jt9HfYOEfiVTJC32yQK_ Waau NxU\#v=onepage \&q=age $\% 20$ and $\% 20$ secong\%20language $\% 20$ acquisition $\& \mathrm{f}=$ false.

Munro, M. y Derwing, T. (1995). Intelligibility: buzzword or buzzworthy? Segunda conferencia anual de procedimientos de pronunciación en el aprendizaje y enseñanza de una lengua extranjera (2010) Estados Unidos. Language Learning. 45. 73-97. Recuperado de http://jlevis. public.iastate.edu/2010\%20Proceedings $\% 20$ 10-25-11\%20-\%20B.pdf.

Muñoz, M. y Valencia, M. (2011). Teaching English Vocabulary to Third Graders Through the Application of the Total Physical Response Method. Pereira: Universidad Tecnológica de Pereira. Recuperado de http://repositorio.utp.edu.co/dspace/bitstream/handle/11059/1940/4281M971. pdf? sequence $=1$ \&isAllowed $=\mathrm{y}$.

Ortega, L. (2009). Understanding Second Language Acquisition. Recuperado de https://books. google.cl/books?hl=es\&lr=\&id=30bKAgAAQB AJ\&oi=fnd\&pg=PP1 \&dq=Ortega + second + lan guage+acquisition\&ots=CbOW_WhOSy\&sig= WZx1HBxCQvrgEAjBG6uYVGFubo\#v=onep age $\& q=$ Ortega $\% 20$ second $\% 20$ language $\% 20$ acquisition $\& \mathrm{f}=\mathrm{false}$.

Richards, J. y Rodgers, T. (2002). Approaches and Methods in Language Teaching. Recuperado de 
https://ia601204.us.archive.org/6/items/ApproachesAndMethodsInLanguageTeaching2ndEditionCambridgeLanguageTeachingLibrary_201610/_Approaches_and_Methods_ in_Language_Teaching__2nd_Edition__Cambridge_Language_Teaching_Library_.pdf.

Rubio, A. y García, I. (2013). El uso de juegos en la enseñanza del inglés en la educación primaria. Revista de Formación e Innovación Educativa Universitaria. 6 (3), 169-185. Recuperado de http://refiedu.webs.uvigo.es/Refiedu/Vol6_3/ REFIEDU_6_3_3.pdf.

Yilorm, Y. (2016). Proceso de enseñanza aprendizaje de la lengua inglesa en escuelas públicas chilenas: ¿Producción o reproducción? Estudios pedagógicos 2016, 42, 103-116. Recuperado de http://www.scielo.cl/scielo.php?script=sci_ arttext\&pid=S0718-0705201600030000.

\section{ANEXOS}

\begin{tabular}{|c|c|c|}
\hline 告 & $\begin{array}{l}\text { UNIVERSIDAD DE CONCEPCION } \\
\text { FACULTAD DE EDUCACION } \\
\text { MAGISTER EN EDUCACION } \\
\text { Profesora: Karin Sepúlveda Navarro }\end{array}$ & 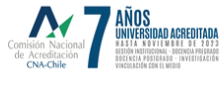 \\
\hline
\end{tabular}

\section{ANEXO 1:}

Test de Pronunciación Oral (Pre-Test y Post-Test de Pronunciación) (Extracto)

\section{Listen and repeat the following words:}

- Eraser

- Sharpener

II. Choose and describe three objects

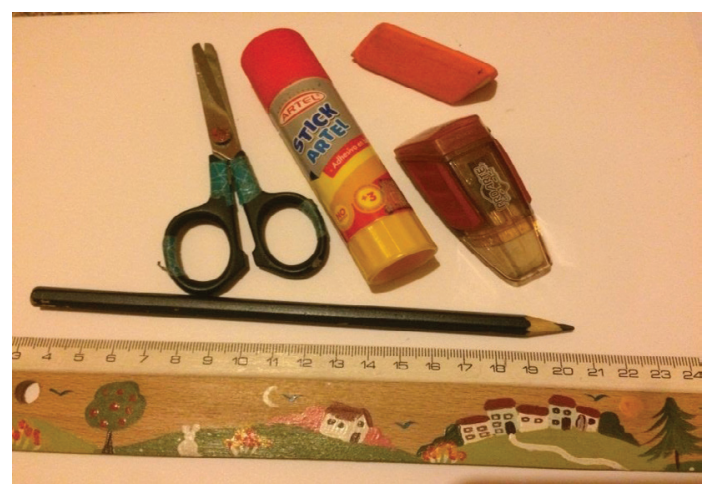

\begin{tabular}{|c|c|c|}
\hline 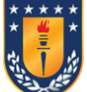 & $\begin{array}{l}\text { UNIVERSIDAD DE CONCEPCION } \\
\text { FACULTAD DE EDUCACION } \\
\text { MAGISTER EN EDUCACION } \\
\text { Profesora: Karin Sepúlveda Navarro }\end{array}$ & 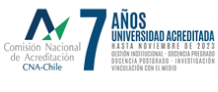 \\
\hline
\end{tabular}

ANEXO 2:

Rúbrica analítica de desempeño de repetición de palabras (extracto)

\begin{tabular}{|c|c|c|c|}
\hline Criterio & $\begin{array}{c}\text { Adecuado } \\
\mathbf{3}\end{array}$ & $\begin{array}{c}\text { Suficiente } \\
\mathbf{2}\end{array}$ & $\begin{array}{c}\text { Insuficiente } \\
\mathbf{1}\end{array}$ \\
\hline $\begin{array}{c}\text { Repeti- } \\
\text { ción de } \\
\text { palabras }\end{array}$ & $\begin{array}{c}\text { Correcta } \\
\text { pronun- } \\
\text { ciación }\end{array}$ & $\begin{array}{c}\text { Uno o dos } \\
\text { errores en } \\
\text { la pronun- } \\
\text { ciación }\end{array}$ & $\begin{array}{c}\text { Tres o más } \\
\text { errores en la } \\
\text { pronuncia- } \\
\text { ción }\end{array}$ \\
\hline
\end{tabular}

\begin{tabular}{|c|c|c|c|c|}
\hline $\begin{array}{c}\text { Palabra } \\
\text { grabada }\end{array}$ & $\begin{array}{c}\text { Pronunciación } \\
\text { del estudiante }\end{array}$ & $\mathbf{3}$ & $\mathbf{2}$ & $\mathbf{1}$ \\
\hline /'’rəIsər/ & & & & \\
\hline /'sa:pnər/ & & & & \\
\hline
\end{tabular}

